\title{
Measurement Models for Survivability and Competitiveness of Very Large E-marketplace
}

\author{
Jingzhi Guo and Chengzheng Sun \\ School of Computing and Information Technology, Griffith University \\ Nathan, QLD 4111, Australian \\ \{J.Guo, C.Sun\}@cit.gu.edu.au
}

\begin{abstract}
E-Marketplace is a man-made profit center with emergent customer requirements. This emergence nature poses many difficulties for e-marketplace to satisfy its customers and thus affects its survivability and competitiveness. To challenge this issue, this paper has introduced a novel emergent emarketplace model, and analyzed and quantified the emergent properties of emarketplace from simple ESMs to very large complex electronic intermediary EIMs. The measurement models developed in this paper are very useful for producing dynamic interaction records appropriate for further simulation model of e-marketplaces.
\end{abstract}

\section{Introduction}

Business-to-business electronic marketplace (e-marketplace) is man-made, which involves costs [8] such as setup cost, maintenance fees, content creation fees, telecommunication link fees and software development fees [10], and needs revenues such as service fees [7] to offset the costs and earn profits for survivals and increasing market competition ability. To achieve this enterprise goal, the best practice is to satisfy customer requirements to deliver the needed services [1], [3], [5], [11].

However, an e-marketplace is an emergent organization, a very large distributed Internet application that supports the mediation of sellers and buyers (simply called as "customers") to finish business deals, in which customers' requirements are in continuous change [2], [6]. This changing nature results in difficulties in capturing customer requirements to design an e-marketplace system [4].

To challenge the above issue, an e-marketplace must be continuously analyzed, measured and dynamically negotiated with customers about emergent requirements. System structure must be flexible to adapt to the incomplete and ambiguous specifications for continuous redevelopment of emergent e-marketplace [12]. Nevertheless, despite the widespread adoption and active roles that e-marketplaces have played, to our best knowledge there is no direct work published on the formal analysis of the emergent e-marketplace requirements for survivability and competitiveness. It is not clear what indicators should be adopted to measure survivability and competitiveness and what properties should be considered to design and simulate an emergent e-marketplace. 
The purpose of this paper, therefore, is to undertake a formal analysis of the requirements for emergent e-marketplaces to quantify the measurements of what are the key factors to the survivability and competitiveness of an emergent e-marketplace.

In the rest of this paper, Section 2 will introduce a novel but generic model of emergent e-marketplace. In Section 3, we will develop the key survivability measurements of a single seller/buyer owned e-marketplace. Section 4 analyzes the key competitiveness measurements of an electronic intermediary e-marketplace. Finally in Section 5, a conclusion will be given and future work will be mentioned.

\section{Emergent E-marketplace}

An emergent e-marketplace is a shared reality in which actors continuously interact with each other to achieve a common goal, lowering business cost and increase revenue but for their own [12]. This shared reality forms the current abstract shape of an e-marketplace and therefore exposes a set of fixed requirements to support the existence of the e-marketplace. These requirements are the "persistent properties (PP)" to satisfy the existence of an e-marketplace. In contrast, the emergent requirements of actors make the e-marketplace in constant negotiation and in continuous change by referencing the original e-marketplace identity. We call these emergent requirements as "emergent properties (EP)", which are the forces to make an e-marketplace fluid.

\subsection{Persistent Properties}

Persistent properties of an e-marketplace can be derived by the analysis of the commonalities and differences between traditional market and e-marketplace. Traditional market is defined as "an arrangement by which buyers and sellers of a commodity interact to determine its price and quantity" [9]. The resemblance between an e-marketplace and a traditional market is that both have products, sellers, buyers, and an arrangement for trading interactions. The difference is the means of how to arrange interaction for sellers and buyers. In traditional market, sellers and buyers interact by face-to-face meeting, verbal or written descriptions of their products to deliver trading information. In e-marketplace, product information is delivered and shared between sellers and buyers in electronic representations via network (generally Internet). Sharing electronic product representations is fundamental to transform a traditional market to an e-marketplace and is the primary task and condition to build an e-marketplace. Although transactional (such as negotiation and payment) and institutional functions (such as legal systems) are often in e-marketplace definition domain [1], they are not the primary task to make e-marketplace alive. So only sellers $S$, buyers $B$, products $P$, electronic product representations $R$, and public accessible network $N$ are considered as persistent properties of an e-marketplace. These persistent properties exist in the lifetime of an emergent e-marketplace.

The persistent properties are footstones of an e-marketplace. Applying for them, we define an e-marketplace as an emergent two-stage functional business organization on Internet. Its first stage functions are about e-matching by forming an 
e-marketplace. Its second stage functions are about e-trading by realizing transactional and institutional functions. E-matching is fundamental to e-trading and has two major tasks: e-representation defined as electronically representing realworld products into machine-readable and multi-firm sharable data, and e-mapping defined as logically mapping sellers' and buyers' electronic product representations into e-tradable forms for trade interactions. In this paper we focus our analysis on the e-matching stage and assume that the profitability of e-trading stage is proportional to the efforts of e-matching stage. So the survivability and competitiveness of an emarketplace is thus dependent on how the first stage tasks are fulfilled.

\subsection{Persistent Relationships}

Persistent properties are not enough to understand how an e-marketplace operates. Only relationships between persistent properties, persistent relationships (PR), reflect the operation of an e-marketplace.

PR 1: Sellers $S$ owns a set of products $S P \subseteq P$, expressed as $S O w n S P$.

PR 2: Buyers $B$ owns a set of products $B P \subseteq P$, expressed as $B O w n B P$.

PR 3: A set of sellers' products $S E P \subseteq S P$ is electronically represented as $S E R$, expressed as SEP RepAs SER.

PR 4: A set of buyers' products $B E P \subseteq B P$ is electronically represented as $B E R$, expressed as BEP RepAs BER.

PR 5: A set of sellers' product representations $S E R$ is displayed on $N$, expressed as SER DispOn $N$

PR 6: A set of buyers' product representations $B E R$ is displayed on $N$, expressed as BER DispOn $N$.

These six relationships are persistent during the lifetime of an e-marketplace. The sum of them defines an e-marketplace.

Definition 1 (Emergent E-marketplace “EEM"): An emergent e-marketplace EEM exists if and only if there exist persistent relationships $P R 1$ and $P R 2$ and $P R 3$ and $P R 4$ and PR5 and PR6.

\subsection{Emergent Properties and Relationships}

Definition 1 provides a static framework and existent conditions of an EEM. To study how an EEM is emergent, we assume that there is a mechanism to make a market arrangement for sellers $S$ and buyers $B$ to interact [9], which is called as interaction mechanism $I$ and releases a dynamic force, originated from sellers $S$ or buyers $B$, acted on network $N$ to have $S$ and $B$ interacting each other. The effects that $I$ generates are the normalized emergent requirements (emergent properties $E P$ ) of customers. The fundamental relationships between EPs (emergent relationship ER) are:

- When an EP generates a set of positive effects, a set of persistent properties (PPs) change themselves in quantity with favorable outcomes for an EEM and further generate a new set of EPs to require EEM to adapt to them. 
- When an $E P$ generates a set of negative effects, a set of $P P$ s change themselves in quantity with unfavorable outcomes for an EEM and further generate a new set of $E P$ s to require the EEM to improve them.

- If the effects that a set of $E P$ s generates lead to any $P P$ disappeared in an EEM such that the quantity of the $P P$ goes to zero, then the EEM is dead.

\subsection{EEM Operational Model}

To describe the interactions between sellers and buyers and to quantify the emergent requirements of an EEM, we define the following interactive relationships:

Definition 2 (Logical Intersection " $\varsubsetneqq$ "): Given three sets of emergent properties $X, Y$ and $I_{\mathrm{xy}}$, then $I_{\mathrm{xy}}=X \mathscr{G} Y$, called $X$ logically intersected with $Y$ at $I_{\mathrm{xy}}$ if and only if (1) $X$ is originated from $S, Y$ is originated from $B$ and $I_{\mathrm{xy}}$ is originated from $I$, and (2) $I_{\mathrm{xy}}$ logically agrees with both at least zero number of $X$ and at least zero number of $Y$.

Definition 3 (Interaction Model): An EEM is said to be interactive if and only if (1) there exists an $I$, and (2) a set $E R$ is generated based on $I_{\mathrm{xy}}=X \mathscr{g} Y$ and $E R \neq \varnothing$.

Emergent relationships are generated in the process of continuous interactions between sellers and buyers. The matched results by interaction mechanism of their emergent requirements become the emergent properties and they further require an EEM to react.

Corollary 1: If $I$ is missing in an EEM, then an EEM generates no EP and is static as Definition 1, then it only provides the e-representation service such as a plain online product catalogue displaying product information.

Corollary 1 shows an interactive EEM is conditional to the existence of $I$. Actually different $I$ qualities lead to different types of EEMs:

Table 1. Types of Electronic Marketplaces

\begin{tabular}{|c|l|l|}
\hline No & \multicolumn{1}{|c|}{ EEM Type } & \multicolumn{1}{c|}{ EEM Owner } \\
\hline 1 & One seller, multiple buyers & Seller \\
\hline 2 & One buyer, multiple sellers & Buyer \\
\hline 3 & Multiple buyers, multiple sellers & Third-party market provider \\
\hline
\end{tabular}

Type 1 and type 2 are most common. Each company website can be regarded as an EEM as it at least provides e-representation service if they do not provide interaction mechanism. Type 3 is the advanced form of type 1 and 2. It aggregates multiple sellers and buyers and runs by a separate firm to serve as an e-marketplace via interaction mechanism.

\section{Survivability Analysis}

In this section, we will first study the simplest e-marketplace, type 1 and 2 of EEMs and refer them as "ESM". To unveil how they survive, we begin by investigating how the emergent properties generated by a seller's or buyer's interaction mechanism $I$ affect their survivability. 


\subsection{Tradability Analysis}

Given an emergent ESM, then its I generates an emergent property - tradability. This part describes how it is generated and affects the ESM's market efficiency.

Definition 4 (Total Marketing Size "TMS"): ESM total marketing size TMS is defined as the sum of SER and BER defined in PR3 and PR4, or expressed as (SER + BER).

$T M S$ reflects an ESM's total market scale at a certain time.

Definition 5 (Tradable Product Representations “ $T$ ”): Given SER and BER defined in PR 3 and PR 4, a set of product representations $T$ is called "tradable" if and only if $T$ $=S E R$ \& BER.

$T$ determines the current exchangeable products between sellers and buyers. It reflects how many electronically represented products are actually tradable.

Definition 6 (Tradability “g”): Given T and TMS defined in definition 4 and 5, the tradability $\mathfrak{J}$ in an ESM is measured as:

$$
\mathfrak{J}=T /(T M S-T)
$$

Tradability measures the capability of an EEM to link sellers and buyers together for trading. It tells how efficient an ESM to satisfy customers' emergent requirements to match the product of sellers or buyers. The ESM's market efficiency measured by $\mathfrak{J}$ is dependent on the capability of interaction mechanism $I$ either provided by sellers or buyers that assists the conversion and mapping of product representations.

\subsection{Profitability Analysis}

A fully efficient ESM may not be necessarily profitable in an emergent e-business environment. For example, the increase of tradability may be increased by decreasing both $T$ and $T M S$ and decrease total profit. As a profit center, an important emergent property - profitability realized at the seller/buyer's interaction mechanism must be introduced to measure and control cost and revenue. To analyze, we assume:

Assumption 1: A piece of electronic product representation $R_{\mathrm{i}} \subseteq T M S$ defined in Definition 4 has an average cost $A C$.

Assumption 2: A piece of electronic tradable product representation $T_{\mathrm{i}}$ defined in definition 5 generates an average revenue $A R$.

Immediately, we have two corollaries:

Corollary 2: If given total marketing size TMS defined in definition 4, then according to Assumption 1, an ESM's total cost $T C=T M S^{*} A C$.

Corollary 3: If given a set of tradable product representations $T$ defined in definition 5, then according to assumption 2, an ESM's total revenue $T R=T^{*} A R$.

These assumptions and corollaries enable us to define profitability.

Definition 7 (Profitability " $\mathcal{P}$ "): Given $T C$ and TR derived from Corollary 2 and 3, the profitability of an ESM is measured as:

$$
\mathscr{P}=(T R-T C) / T R
$$

Corollary 4: If profitability $\mathcal{P} \leq 0$, then an ESM is in difficulty to survive. If profitability $\mathscr{P}>0$, then an ESM is in a favorable survival position. If $\mathscr{P}$ goes to 1 , then 
the cost of an ESM goes to 0 and the ESM tends to be frictionless like the traditional market assuming that market is efficient and no informational cost.

Obviously, the profitability of ESM is positively proportional to the tradable product representations $T$ and negatively proportional to the total market size TMS. However high profitability may not contribute to improving tradability if both TMS and $T$ remain constant but the unit revenue of $T$ is increased when a seller or buyer adopts a radical profit strategy or the ESM tends to be frictionless.

\subsection{Survivability Analysis}

The analysis of Section 3.1 and 3.2 shows the tradability and profitability may independently increase or decrease. It suggests that we must further develop survivability to include the joint effects of the above two emergent properties.

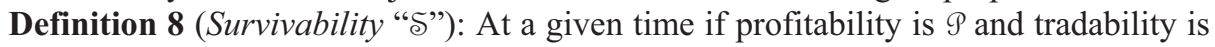
$\mathcal{J}$, then the survivability is.

$$
\Im=\mathfrak{P} * \mathfrak{J}
$$

Survivability reflects at a given time the quality of an ESM in terms of e-market efficiency and profits. To further analyze the dynamic effects of tradability and profitability, we gather a time series of survivability data to reflect the changes of an ESM's survivability.

Definition 9 (Market Growth Rate "G"): If from time $t_{\mathrm{a}}$ to $t_{\mathrm{b}}$, survivability is from $\varsigma_{\mathrm{a}}$ to $\varsigma_{\mathrm{b}}$, then the market growth rate is:.

$$
\mathcal{G}=\left(\varsigma_{\mathrm{b}}-\varsigma_{\mathrm{a}}\right) /\left(\mathrm{t}_{\mathrm{b}}-\mathrm{t}_{\mathrm{a}}\right)
$$

Market growth rate reflects the ability of an ESM to cope with the long-run emergent ESM requirements.

Corollary 5: If $\mathcal{G}$ is positive, an ESM is in a favorable survival condition. If $\mathcal{G}$ is negative, an ESM is in an unfavorable survival condition.

In summary, an ESM's survivability depends on the emergent requirements of tradability and profitability. However, it is worth to note that the live of an ESM may be independent of survivability in that a seller/buyer may only want to benefit from the e-representation to display products as promotional purpose.

\section{Competition Analysis}

Emergence theory assumes an organization is in the state of being continual process, never arriving but always in transition [12]. Coincidently, economic theories prove that changes of market power between sellers and buyers change the competition power and market type [9]. Often competition power can be obtained by reconstructing market types by alliance. Applying these theories, this section will analyze the competitiveness of type 3 e-marketplace evolved from a set of ESMs. 


\subsection{Electronic Intermediary Marketplace}

Drives of E-intermediary Marketplace. The ESM described in Section 3 is a typical EEM under perfect competition where all sellers and buyers are responsible for their own e-matching service and fully compete with each other. It is often presented as a website such as a standalone electronic catalogue or a corporate website with embedded catalogue [10] such as GE Polymerland ${ }^{1}$. It is comparatively small and has no enough power to exert influences on selling or buying. Its customers are limited to the related partners in a narrow industry or random product seekers. From the viewpoint of all TMSs of ESMs, an individual ESM is insignificant in market share because its product information is difficult to reach to and share by all customers in emarketplaces. To attract more customers, one choice is to aggregate multiple ESMs into an electronic intermediary marketplace (EIM) representing a superset of some ESMs, in which a larger set of sellers and/or buyers map their electronic product representations via brokering, mediation, federation and mapping. A practical example is CommerceOne ${ }^{2}$ who provide third-party B2B marketplaces. This choice is a natural evolution of the ESM development. The benefits of adopting an EIM are:

- Aggregating a set of individual ESMs to increase competition power.

- Reaching some consensus to adopt a unified product representation standard for all its sellers and buyers to increase tradability. The proper standards enable an EIM to compete with those un-joined sellers, buyers and other EIMs.

- Integrating the increased number of de facto product standards, international standards and ad-hoc electronic product representations.

EIM Persistent Properties and Relationships. As a superset of some ESMs, an EIM has its special persistent properties that are: an EIM provider who represents a certain set of sellers and buyers, and a set of mediating product representations (IER) that mediates sellers' and buyers' product representations SER and BER defined in PR3 and PR4. So the new persistent relationships in EIM are:

PR 7: Some sellers' SER is mediated via a set of mediating product representations $I E R$ provided by an EIM provider, expressed as SER MedBy IER.

PR 8: Some buyers' $B E R$ is mediated via a set of mediating product representations IER provided by an EIM provider, expressed as BER MedBy IER.

PR 9: A set of product representations IER is owned by an EIM provider, expressed EIM Own IER.

PR 10: A set of product presentations $I E R$ is displayed on network $N$, expressed as IER DispOn $N$.

Definition 10 (Electronic Intermediary Marketplace "EIM"): An electronic intermediary marketplace EIM exists if and only if there exist persistent relationships PR1, PR2, PR3, PR4, PR7, PR8, PR9 and PR10.

Unlike an ESM, an EIM acts as an independent interaction mechanism. The relationships existed in an EIM reflect the existence conditions of an EIM.

$1 \mathrm{http}: / / \mathrm{www} . \mathrm{gepolymerland.com}$

2 http://www.commerceone.com 


\subsection{EIM Mediability}

Similar to tradability in an ESM, the emergent property, mediating ability, for sellers' and buyers' product representations is important to an EIM's survival and should be accurately defined.

Definition 11 (Target Mediating Volume "TMV"): Given SER and BER defined in PR 3 and PR4, a set of product representations $T M V$ is called "target mediating volume" if and only if there exist two sets of product representations $M S R$ and $M B R$ such that $M S R \subseteq S E R$ and $M B R \subseteq B E R$, and $T M V=M S R+M B R$.

$M S R$ and $M B R$ are sellers' and buyers' electronic product representations ready to be mediated in an EIM. The sum of them is the target volume for an EIM to mediate. It must be noted that the quantity of $S E R$ and/or $B E R$ in an EIM may have increased compared with that in an ESM where SER/BER is from only one seller/buyer if this ESM is a seller's or buyer's EEM.

Definition 12 (Mediable Representations “M"): Given $M S R$ and $M B R$ defined in Definition 11, IER defined in PR 9, a set of product representations $M$ is said to be "mediable" if $M=M S R \mathscr{G}$ IER $\cap I E R ~ 9 M B R$.

The volume of mediable product representations indicates how many e-mapping services an EIM can provide to satisfy its sellers and/or buyers. It reflects the fulfillment of the target market.

Definition 13 (Mediability "Or"): Given $T M V$ and $M$ defined in definition 11 and definition 12 , the mediability of $\mathscr{K}$ is measured as:

$$
\text { ๑ }=\mathrm{M} /(T M V-M)
$$

Mediability reflects an EIM's market efficiency about its target market. An EIM's profitability, survivability and market growth rate are similar to ESM. The indicators can be directly given as:

Definition 14 (EIM Profitability " $₫ 99$ ", EIM Survivability " $9 \subseteq "$ and EIM Market Growth Rate " $9 \mathcal{G}$ "): If given $T M C=T M V^{*} A C$ and $T M R=M^{*} A R$ derived from Assumption 1, 2 and definition 11 and 12, then:

$$
\begin{aligned}
& \mathscr{् P}=(T M R-T M C) / T M R \\
& 9 \Im=\Im \mathfrak{P} * \text { 丹 } \\
& \mathscr{G}=\left(\mathscr{S}_{\mathrm{b}}-\mathscr{S}_{\mathrm{a}}\right) /\left(t_{\mathrm{b}}-t_{\mathrm{a}}\right)
\end{aligned}
$$

The above indicators jointly describe the long-run survivability of an EIM.

\subsection{EIM Competition Power}

To completely reflect the competitiveness of an EIM in an emergent EEM, we should compare with other EIMs. We compare survivability between EIMs to determine an EIM's competition power. 
Definition 15 (EIM Competition Power "ẹ"): Given a set of $E I M={ }_{\mathrm{d}}\{0 \leq i, j \leq \mathrm{n} \mid$ $\left.E I M_{\mathrm{i}, \mathrm{j}}\right\}$ defined in Definition 10, and a set of $E I M$ survivability $9 \subseteq{ }_{\mathrm{d}}\{0 \leq i, j \leq \mathrm{n} \mid$ $\left.\Im \varsigma_{i, j}\right\}$ defined in Definition 14(2), then:

$\mathrm{EIM}_{\mathrm{i}}$ 's relative competition power against $\mathrm{EIM}_{\mathrm{j}}$ is:

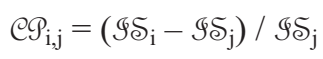

EIMi's absolute competition power against the whole set of EIM is:

$$
\mathfrak{C P}_{\mathrm{i}}=\left(\mathscr{S S}_{\mathrm{i}}-\mathfrak{9 S}\right) / \mathfrak{S S}
$$

Relative competitive power compares an EIM with another EIM. Absolute competitive power compares an EIM with the whole intermediary provider market. A positive figure means a strong market competition power to attract sellers and buyers to join in.

Corollary 6: For any two EIMi and EIMj, if any $\mathcal{E S i}_{\mathrm{i} j}$ is approximate to 0 , the overall EIM marketplace goes to perfect competition.

Corollary 7: If there exist one $E I M_{\mathrm{i}}$ such that $\mathcal{E S}_{\mathrm{i}, \mathrm{j}}$ is always approximate to 1 against $E I M_{\mathrm{j}}, E I M_{\mathrm{i}}$ goes to be monopolistic in the overall EIM marketplace.

Corollary 7 and 8 measures the market power of an EIM over the sellers, buyers and other EIMs, which changes e-marketplace types.

\subsection{Competitive Analysis}

The above sections have described the changes of an EIM's competition power. But how to increase EIM competition power is still not clear. This section, we will analyze the changing relationships between emergent properties to find out the solution. As Definition 14, EIM profit is determined by the factors of $T M R_{\mathrm{EIM}}$ $=M^{*} A R_{\mathrm{EIM}}$ and $T M C_{\mathrm{EIM}}=T M V^{*}\left(A C_{\mathrm{EIM}}+A C_{\mathrm{SB}}\right)$. So an EIM's total profit is $T M R_{\mathrm{EIM}}-$ $T M C_{\mathrm{EIM}}$ and the target is to either increase $M$ and $A R_{\mathrm{EIM}}$, or decrease $A C_{\mathrm{EIM}}+A C_{\mathrm{SB}}$. For the total customers of the EIM, if, for each mediable product representation, it can gain $A R_{\mathrm{SB}}$, then its profit from using the EIM is $M^{*} A R_{\mathrm{SB}}-T M V^{*} A C_{\mathrm{SB}}$. Obviously, $A R_{\mathrm{EIM}}$ partially and proportionally determines $A C_{\mathrm{SB}}$ in that part of $A C_{\mathrm{SB}}$ is the $A R_{\mathrm{EIM}}$. For example, EIM increases service fees to increase $A R_{\text {EIM }}$ but it also increases the cost of customers' $A C_{\mathrm{SB}}$. Another part of $A C_{\mathrm{SB}}$ is the sinking cost of sell customers who electronically represent their products by themselves. Therefore the limit for an EIM to increase $A R_{\text {EIM }}$ is that the decreasing total profit of customers is still positive and the conditions that other EIMs have not provided customers better profit rates. Otherwise customers will either exit from the EIM or turn to other EIMs. Another method to increase profit is to decrease TMV, but it is not under an EIM's control, customers may increase product representation volume hence increase their emarketplace sinking cost but still try to find a better EIM to absorb it. Further method is to decrease $A C_{\mathrm{EIM}}$ but it may hinder an EIM to provide better e-mapping service due to the less input of the development fees. The obvious better solution is to increase $M$, which has no limit and has the greatest potential. To increase volume $M$ is to map more sellers' and buyers' electronic product representations. 


\section{Conclusion}

By introducing a novel emergent e-marketplace model, this paper has formally analyzed the emergent properties of simple ESMs and large and complex EIMs. Concomitantly, it has quantified the key measurements of survivability and competitiveness of a very large e-marketplace. As an analysis result, it points out that the key to adapt to the customers' emergent requirements is to increase mediability. Satisfying this requirement can keep an EIM in a favorable market position with high survivability and strong competition power and obtain a win-win outcome. Measurement models developed in this paper have many implications in which the most direct is to apply these models to simulation models of very large emarketplaces. The changing values of emergent properties over time are appropriate interaction records for simulation. The future work includes to explore the appropriate electronic representation of products and to further develop measurement model for etrading of the second stage e-marketplace.

\section{References}

1. Bakos, Y., The Emerging Role of Electronic Marketplaces on the Internet, Communications of the ACM, Vol. 41/No. 8, August 1998, 35-42.

2. Damsgaad, J., and Truex, D., Binary Trading Relations and the Limits of EDI Standards: The Procrustean Bed of Standards, European Journal of Information Systems, 2000, 9(3) 173-188.

3. Hoffner, Y., Facciorusso, C., Field, S., and Schade, A., Distribution Issues in the Design and Implementation of a Virtual Market Place, Computer Networks, 32 (2000) 717-730.

4. Maidantchik, C., Montoni, M., and Santos, G., Learning Organizational Knowledge: An Evolutionary Proposal for Requirements Enginnering, in Proceeding of ACM SEKE'02, July 15-19, 2002, Ischia, Italy, 151-157.

5. Malone, T. W., Yates, J., and Benjamin, R., Electronic Markets And Electronic Hierarchies, Communications of the ACM, Vol. 30/No. 6, June 1987, 484-497

6. Ngwenyama, O. K., Groupware, social action and organizational emergence: on the process dynamics of computer mediated distributed work, Accounting, Management and Information Technology, 8 (1998) 127-146.

7. Phillips, C. and Meeker, M., Collaborative Commerce, The B2B Internet Report, Morgan Stanley Dean Witter, April 2000.

8. Rose, M., Implications of Costly Information, The Library of Economics and Liberty, April 22, 2002, http://www. econlib.org/library/Columns/Teachers/information.html.

9. Samuelson, P.A., Nordhaus, W. D., Richardson, S., Scott, G., and Wallace, R., Economics Volume 1 Microeconomics, Third Australian Edition, McGraw-Hill Book Company Sydney, 1992.

10.Segev, A., Wan, D., and Beam, C., Electronic Catalogs: a Technology Overview and Survey Results, in proceedings of $A C M$ CIKM'95, Baltimore, MD, USA, 1995, 11-18.

11.Strader, T., and Shaw, M., Characteristics of Electronic Markets, Decision Support Systems, 21 (1997) 185-198.

12.Truex, D. P., Baskerville, R., and Klein, H., Growing Systems in Emergent Organizations, Communications of the ACM, Vol. 42/No. 8, August 1999, 117-123. 